\title{
Configuración del Grado en Ingeniería de Organización Industrial en las universidades españolas
}

\section{Configuration of the Bachelor's Degree in Industrial Engineering at Spanish Universities}

\author{
Josefa Mula, Manuel Díaz-Madroñero y Raúl Poler
}

Departamento de Organización de Empresas, Centro de Investigación Gestión e Ingeniería de Producción (CIGIP), Universitat Politècnica de València, Escuela Politécnica Superior de Alcoy, Plaza Ferrándiz y Carbonell, 2, 0380 I, Alcoy-Alicante. España. fmula@doe.upv.es, fcodiama@doe.upv.es,rpoler@doe.upv.es

Fecha de recepción: 5-4-2011

Fecha de aceptación: 29-7-2011

Resumen: El objetivo principal de este trabajo es analizar la configuración de la titulación del Grado en Ingeniería de Organización Industrial (GIOI) en las universidades españolas. El núcleo central de este trabajo se basa en examinar los antecedentes históricos de esta titulación y analizar su estado actual en las universidades españolas tras el proceso de convergencia europea. Además de mostrar el mapa actual de la titulación se comparan los planes de estudios propuestos. Con ello, se pretende estructurar el conocimiento de esta titulación para que pueda servir de referencia a docentes, investigadores y usuarios de la misma para el desarrollo de sus actividades. También, podría servir de punto de partida o modelo para su consideración, cuando llegado el momento oportuno, una universidad decida implantar dicha titulación.

Palabras Clave: diseño, implantación, titulación, grado en ingeniería de organización industrial, universidades españolas.

Abstract: The main objective of this work is to analyse the configuration of the bachelor's degree in industrial engineering (GIOI) at Spanish universities. The central focus is based on the review of the historical antecedents of this degree, since the 19th century, and the analysis of its current status at the Spanish universities after the European convergence process. The main goal of the $\mathrm{GIOI}$ is to train professionals capable of performing tasks of analysis, evaluation, design and management in engineering organization within four main approaches: operations management, economic analysis, management and organizational strategic management, and with technology training in the areas of automatic, construction, electronics, mechanics, chemistry, environmental and manufacturing process technologies. Furthermore, the input profiles are stated and the output profiles of the GlOI are defined establishing general competencies or knowledge, transversal competencies or abilities and specific competencies or skills. The recommendations provided by ADINGOR (Association for the development of Industrial Engineering, Spain) have been reviewed with regard to the requirements for the verification of the GIOI programme by ANECA (The National Agency for Quality Assessment and Accreditation of Spain). ADINGOR proposed a reference curriculum, at subject level, consisting of: basic courses, industrial technologies, information technologies, quantitative methods and production management, business management and economic engineering and industrial and technological environment. Besides showing the current map of this degree, the proposed study programmes are compared. At present, there are 23 schools 22 Spanish universities that offer, have been verified by ANECA or are proceeding to the verification of the GIOI.The GIOI has a major presence in the Spanish territory in those regions with a considerable industrial tradition. Almost the totality of the universities that offer the $\mathrm{GIOI}$ provide public access to the syllabus on the Internet, which allowed his consultation and analysis. We have analyzed the available syllabi of I 5 university centres and we have compared the general structure of the curricula and the specific courses offered. In summary, the curricula consist of 60 ECTS of basic courses with the majority of credits assigned to mathematics, physics and chemistry; an average of 45.7 ECTS for industrial technologies, with the majority of credits given to mechanical and materials technology, energetic theory and mechanical of fluids and automatic and electronics; and an average of 69.7 ECTS related to specific learning of industrial organization, with the majority of credits given to quantitative methods, production management and strategic management. The balance of the courses are related to general economics, business administration, logistics, work management, financial management, information systems, marketing, quality and project management. For typologies of courses, the majority of credits go to PRO (production management, work management and logistic) + QM (quantitative methods), which match with the ADINGOR's proposal. The main contribution of this paper is to provide a reference structure of the GIO degree for academics, researchers and practitioners. Spanish universities could use the proposed reference curriculum as a starting point when deciding to implant the bachelor's degree in industrial engineering (GIOI).

Key words: Design, implantation, degree, bachelor's degree in industrial engineering, Spanish universities. 


\section{Introducción}

En un momento de cambios importantes como el que vive actualmente la universidad española en lo relativo a la definición de sus títulos, resulta de gran interés analizar los recientes planes de estudios de los grados que se imparten en varias universidades para constatar sus similitudes y diferencias y, en su caso, proporcionar elementos de decisión a las universidades que deseen implantar dichos grados en el futuro. El objetivo de este artículo es analizar la configuración actual de la titulación de Grado en Ingeniería de Organización Industrial (GlOl) estudiando ampliamente la organización y contenido de la misma en todos los centros universitarios españoles donde se imparte. Para ello, se abordan en primer lugar los antecedentes históricos de la titulación de GIOI. A continuación, se analiza de forma global la titulación: los objetivos, el perfil del ingresado y del egresado y cómo se oferta actualmente dicha titulación en el territorio nacional. Seguidamente, se comparan la estructura general y las asignaturas propuestas en los planes de estudios del GIOl impartidos en las universidades españolas.

La contribución principal de este artículo es un estudio actualizado de la situación de la titulación de GIOI en el contexto universitario español. Este estudio podrá servir de referencia para la propuesta o mejora de planes de estudios de esta titulación en el contexto actual y en el futuro inmediato. También, como punto de partida para nuevos investigadores o usuarios de la disciplina.

El artículo se ha organizado como sigue. En primer lugar, en la Sección 2 se resumen los antecedentes históricos del GIOI. En la Sección 3 se describen las características generales del GIOI. En la sección 4 se analizan los planes de estudios de la titulación del GIOI en las universidades españolas. Finalmente, en la Sección 5 se exponen las conclusiones.

\section{Antecedentes históricos del Grado en Ingeniería de Organización Industrial}

En I 886, H.R. Towne presenta en la ASME (American Society of Mechanical Engineers) una comunicación titulada «The engineer as economist» donde propone que la gestión de plantas de fabricación es tan importante como su ingeniería.
En 1908 se crean en Penn State University los estudios de Ingeniería Industrial (Industrial Engineering), que adoptan como núcleo central las enseñanzas de Frederick W. Taylor relativas a la organización científica del trabajo.

En 1955 se crea en España la primera Escuela de Organización Industrial dependiente del Ministerio de Industria para impartir enseñanzas en forma de cursos de postgrado. En 1964 se crea la Ingeniería en Organización Industrial como una especialidad más de los estudios de Ingeniería Industrial.

En 1979, la UNESCO en su documento Formation des Ingenieurs et environment:Tendentes et perspectivas define al Ingeniero de Gestión como: «Forma parte de un equipo en el que se encuentran los cuadros comerciales, economistas, financieros, estadísticos, psicólogos, etc. Participa de la gestión científica de los negocios, realiza estudios de mercado y puede acceder a los altos niveles de dirección de la empresa si además de su formación técnica posee una formación alta en administración».

La titulación de segundo ciclo de Ingeniería de Organización Industrial (IOI) se estableció en el Real Decreto 1401/1992, de 20 de noviembre (Boletín Oficial del Estado número 306, de 22 de diciembre de 1992). El objetivo central de este título es formar profesionales capaces de desempeñar labores de análisis, evaluación, diseño y gestión en el área de conocimiento de organización de empresas y dentro de sus 4 enfoques principales: la Dirección de Operaciones, el Análisis Económico, la Gestión Organizativa y la Dirección Estratégica. Además, los titulados reciben una formación tecnológica en las áreas de automática, construcción, electricidad, electrónica, mecánica, química, tecnologías del medioambiente y tecnologías de los procesos de fabricación. Las directrices generales de esta titulación indican que se trata de enseñanzas de sólo segundo ciclo, con una duración de 2 años y una carga lectiva mínima de 120 créditos. En la actualidad, en España hay 27 centros universitarios que imparten el título de segundo ciclo de $\mid \mathrm{Ol}, 7$ centros privados y 20 centros públicos, de 21 universidades españolas, 7 universidades privadas y 14 universidades públicas. Se observa que los planes de estudios son relativamente recientes, siendo el más antiguo del año 1992 (fecha en la que se estableció como tal la titulación de 101 en España) y el más reciente de 2002. Esta titulación de segundo ciclo matriculará alumnos de nuevo ingreso previsiblemente hasta el curso académico 2012/2013. No obstante, los estudios de $1 \mathrm{OI}$ continuarán en las uni- 
versidades españolas como titulaciones de grado y/o máster. Las titulaciones de grado se centrarán en la formación general de los ingenieros de organización industrial tanto desde la perspectiva de tecnologías industriales como de la de organización industrial. Por otro lado, los estudios de máster tendrán como objetivo principal la especialización de los estudiantes en cualquiera de los enfoques principales de la organización industrial. Este artículo se centra en los estudios de GIOI.

El lector puede ampliar la información anterior en ANECA (2006) y Figuera (2007).

\section{El Grado en Ingeniería de Organización Industrial}

En el curso académico 2010/20 I I, y en el marco del Espacio Europeo de Educación Superior (EEES), se han implantado en España los estudios de GIOI, cuyos planes de estudios verificados o en trámite por la ANECA, se abordan a lo largo de este artículo. Como consecuencia de la Declaración de Bolonia (1999) y del Real Decreto I393/2007, por el que se establece la ordenación de las enseñanzas universitarias oficiales, y $861 / 2010$, por el que se modifica el Real Decreto 1393/2007, cada universidad tiene la posibilidad de elaborar sus propios planes de estudios dentro del marco normativo existente.

\section{I. Objetivos de la titulación}

El objetivo central del título de GlOl es formar profesionales capaces de desempeñar labores de análisis, evaluación, diseño y gestión en el área de ingeniería de organización y dentro de cuatro enfoques principales: la dirección de operaciones, el análisis económico, la gestión organizativa y la dirección estratégica. Además, los titulados reciben una formación tecnológica en las áreas de automática, construcción, electricidad, electrónica, mecánica, química, tecnologías del medioambiente y tecnologías de los procesos de fabricación. Para los autores de este artículo, los conocimientos, capacidades y destrezas del graduado en ingeniería de organización industrial se pueden resumir en los siguientes objetivos:

a) Formar profesionales capaces de concebir, organizar y gestionar empresas de producción y servicios, así como otras instituciones en todas sus áreas funcionales y dimensiones: operaciones, económica, organizativa, estratégica y técnica, con una fuerte dimensión emprendedora y de innovación. b) Facultar profesionales competentes para asesorar, proyectar, hacer funcionar, mantener y mejorar sistemas, estructuras, instalaciones, sistemas de producción, procesos, y dispositivos con finalidades prácticas, económicas y financieras.

c) Proporcionar una visión integral de la organización tanto desde el punto de vista estratégico al operativo para toda la cadena de valor orientada hacia la calidad total.

d) Valorar la importancia de la gestión de la tecnología como factor clave para la mejora de la competitividad en el entorno actual.

e) Capacitar profesionales para gestionar, evaluar y mejorar sistemas de información basados en tecnologías de la información y las comunicaciones.

f) Proporcionar bases sólidas en ingeniería de organización (dirección de operaciones, análisis económico, gestión organizativa, dirección estratégica) y tecnología.

g) Promover las capacidades y competencias dirigidas hacia la resolución de problemas, la iniciativa, la toma de decisiones, la creatividad, el análisis y el razonamiento crítico.

h) Capacitar al egresado en un conjunto de competencias sociales, interpersonales, emocionales y de trabajo en un entorno multidisciplinar.

i) Transmitir al egresado una actitud respetuosa con las personas, la seguridad en el trabajo, el entorno social y ambiental, basada en la cultura de la mejora continua, formación e innovación.

j) Habilitar al egresado de destrezas técnicas y de una sensibilización que le permita impulsar, organizar y llevar a cabo mejoras e innovaciones tanto en procesos, bienes y servicios.

k) Dotar a los egresados de una actitud proactiva y emprendedora que les facilite expandir las organizaciones para las que trabajen con nuevas líneas de actuación o crear su propia empresa.

1) Proporcionar las bases necesarias para el aprendizaje autónomo, o para cursar estudios de postgrado y/o doctorado que le permitan profundizar y/o especializarse en los diferentes campos de la ingeniería de organización industrial.

Para otras propuestas de la definición de los objetivos y las competencias del título de GIOI se refe- 
rencia al lector a la Declaración de Bolonia (1999), Marín-García et al. (2008) y Marín-García et al. (20 I0). También cabe resaltar el análisis de las competencias profesionales requerido al ingeniero que se presenta en Solana (2009).

\subsection{Perfil de los ingresados a la titulación}

En lo relativo a los requisitos de acceso a los estudios conducentes a la obtención del título de GIOI, éstos son los establecidos con carácter general para el acceso a las enseñanzas universitarias oficiales de grado en el capítulo I del Real Decreto 1892/08. En el caso de las pruebas de acceso a la universidad (PAU), puesto que estos estudios se enmarcan dentro de la rama de conocimiento de ingeniería y arquitectura, los estudiantes de $2^{\circ}$ de bachillerato deberían cursar las materias indicadas en el Anexo I del Real Decreto 1892/08 de la modalidad de ingeniería y arquitectura. Los títulos de técnico superior de formación profesional de la rama de conocimiento de ingeniería y arquitectura indicados en el Anexo II del Real Decreto I892/08 también son conducentes al GIOI. Otras vías de acceso tales como: técnico deportivo superior, pruebas de acceso a mayores de 25 años, mayores de 40 años que acrediten experiencia profesional y laboral, mayores de 45 años, titulados universitarios y/o estudiantes procedentes de sistemas educativos superiores pueden consultarse en el Real Decreto 1892/08.

\subsection{Perfil de los egresados de la titulación}

Para el diseño de un nuevo título de grado se debe partir de las competencias requeridas o del perfil de egresado para definir las materias de formación básica, obligatorias y optativas del plan de estudios. Este apartado tiene como objeto definir las características generales del perfil del egresado de la titulación de GIOI. Este análisis dará como resultado una tabla de competencias' en términos de conocimientos, capacidades y destrezas deseables. Obviamente, en función de las asignaturas obligatorias y optativas que cada universidad adopte en sus planes de estudios se generarán particularidades concretas en los perfiles de sus egresados.

En el documento-marco sobre la integración del sistema universitario español en el EEES (Ministerio de Educación, Cultura y Deporte 2003) se expone «los objetivos formativos de las enseñanzas oficiales de nivel de grado tendrán, con carácter general, una orientación profesional, es decir, deberán proporcionar una formación universitaria en la que se integren armónicamente las competencias genéricas básicas, las competencias transversales relacionadas con la formación integral de las personas y las competencias más específicas que posibiliten una orientación profesional que permitan a los titulados una integración en el mercado de trabajo». Además dice, «estas titulaciones deberán diseñarse en función de unos perfiles profesionales con perspectiva nacional y europea de unos objetivos que deben hacer mención expresa de las competencias genéricas (conocimientos), transversales (capacidades) y específicas (destrezas) que pretenden alcanzar»». Existen otras clasificaciones de competencias en el ámbito de la educación superior, tales como las usadas en el Proyecto Tuning (Maffioli y Augusti 2003), clasificación de Lasnier (2000), clasificación de Barnett (200 I), clasificación de Universidad de Deusto (2000), etc. En este documento se utilizará la clasificación de competencias del documento-marco del Ministerio de Educación, Cultura y Deporte (2003).

Para el GIOI se considera necesaria la misma formación básica que para los grados que habiliten para el ejercicio de la profesión de Ingeniero Técnico Industrial (Grado en Ingeniería Química, Grado en Ingeniería Mecánica, Grado en Ingeniería Eléctrica, Grado en Ingeniería Electrónica y Grado en Ingeniería Textil) según la Orden CIN/35I/2009, de 9 de febrero, del BOE Núm. 44 de 20 de febrero de 2009.

Con respecto a las materias obligatorias, las competencias genéricas o conocimientos a adquirir se han clasificado en conocimientos de ingeniería de organización y tecnológicos según la Tabla I.

\footnotetext{
' El significado de estas palabras corresponde a las siguientes acepciones del Diccionario de la Real Academia de la Lengua Española (RAE):
}

Destreza: Habilidad, arte, primor o propiedad con que se hace algo.

Capacidad: Aptitud, talento, cualidad que dispone a alguien para el buen ejercicio de algo.

Competencia: Pericia, aptitud, idoneidad para hacer algo o intervenir en un asunto determinado.

Conocimiento: Acción y efecto de conocer.

Conocer: Averiguar por el ejercicio de las facultades intelectuales la naturaleza, cualidades y relaciones de las cosas. 
En el diseño del GIOI habría que relacionar las diferentes materias obligatorias y las competencias genéricas o conocimientos de la Tabla I que se pretende que alcancen los estudiantes con el aprendizaje de dichas materias.

Las competencias transversales o capacidades hacen referencia a los atributos que deberían tener los graduados en ingeniería de organización industrial y están básicamente referidas a la capacidad organizativa y de relación del individuo con su entorno. Las competencias transversales o capacidades se han subdivido en instrumentales, personales y sistémicas:

- Instrumentales: Corresponden a las habilidades cognoscitivas (capacidad de comprender y manipular ideas y pensamientos), metodológicas (capacidad organizativa, estrategias, toma de decisio- nes y resolución de problemas), tecnológicas y lingüísticas.

- Personales: Se refieren a la interacción social y cooperación del titulado con su ámbito social: capacidad de exteriorizar los propios sentimientos, habilidad crítica y autocrítica.

- Sistémicas: Capacidades o habilidades de visión y análisis de realidades totales y multidimensionales: corresponden a los sistemas como un todo.

Las competencias transversales o capacidades consideradas se muestran en la Tabla 2.

En el diseño del GIOI habría que relacionar las diferentes materias obligatorias y las competencias transversales o capacidades de la Tabla 2 que facilitan su aprendizaje.

Tabla 1

Competencias genéricas o conocimientos

\begin{tabular}{ll}
\hline \multicolumn{1}{c}{ Conocimientos de ingeniería de organización } & \multicolumn{1}{c}{ Conocimientos tecnológicos } \\
\hline 01. Comercialización y marketing & 11. Automática \\
02. Contabilidad y finanzas & 12. Construcción \\
03. Dirección de operaciones & 13. Electricidad \\
04. Economía industrial & 14. Electrónica \\
05. Estadística & 15. Mecánica \\
06. Estudio del trabajo & 16. Química \\
07. Gestión de proyectos & 17. Tecnologías del medioambiente \\
08. Métodos cuantitativos & 18. Tecnologías del proceso \\
09. Sistemas de información & \\
10. Teoría estratégica de la empresa & \\
\hline
\end{tabular}

Tabla 2

\section{Competencias transversales o capacidades}

\begin{tabular}{|c|c|c|}
\hline Capacidades instrumentales & Capacidades personales & Capacidades sistémicas \\
\hline $\begin{array}{l}\text { 19. Capacidad de análisis y síntesis } \\
\text { 20. Capacidad de gestión de la } \\
\text { información } \\
\text { 21. Capacidad de organización y } \\
\text { planificación } \\
\text { 22. Capacidad de tomar decisiones } \\
\text { 23. Capacidad para analizar y buscar } \\
\text { información proveniente de fuentes } \\
\text { diversas } \\
\text { 24. Capacidad para la resolución de } \\
\text { problemas }\end{array}$ & $\begin{array}{l}\text { 25. Conocimientos de las TIC relativas al } \\
\text { ámbito de estudio } \\
\text { 26. Capacidad crítica y autocrítica } \\
\text { 27. Capacidad para trabajar en equipo } \\
\text { 28. Compromiso ético en el trabajo } \\
\text { 29. Habilidades en las relaciones } \\
\text { interpersonales } \\
\text { 30. Trabajar en entornos de presión } \\
\text { 31. Trabajo en equipo de carácter } \\
\text { multidisciplinar } \\
\text { 32. Habilidades directivas }\end{array}$ & $\begin{array}{l}\text { 33. Capacidad de adaptación a nuevas } \\
\text { situaciones } \\
\text { 34. Capacidad de aprendizaje autónomo } \\
\text { 35. Creatividad } \\
\text { 36. Iniciativa y espíritu emprendedor } \\
\text { 37. Liderazgo } \\
\text { 38. Sensibilidad hacia temas } \\
\text { medioambientales }\end{array}$ \\
\hline
\end{tabular}


Las competencias específicas o destrezas que se propone que obtengan los graduados en ingeniería de organización industrial se muestran en la Tabla 3 subdivididas en dos áreas principales: destrezas para la organización y destrezas tecnológicas.

A partir de este momento, ya se estaría en disposición de definir apropiadamente el perfil del egresado del título de GIOI. Este grado capacita al egresado para la ingeniería de organización de empresas industriales y de servicios, así como instituciones de diversa índole (empresas privadas, administración pública, Universidad, empresas consultoras, organizaciones sin ánimo de lucro, etc.) y, principalmente, en las siguientes áreas: comercialización y marketing, contabilidad y finanzas, dirección estratégica, dirección de operaciones, organización del trabajo y factor humano, gestión de la innovación, gestión de proyectos, tecnologías de proceso y sistemas de información. En general, el graduado en ingeniería de organización industrial debería adquirir el carácter de un experto, una persona práctica, con habilidades claras, experimentada en su campo, que pueda abordar problemas de análisis, diseño y gestión con criterios profesionales y con el manejo de herramientas técnicas. Para ello debe ser capaz de:

- Contribuir a la adecuada gestión de la asignación de recursos. Identificar y anticipar problemas al respecto.

- Establecer sistemas para la toma decisiones en base al análisis económico y financiero.

- Elaborar sistemas para la planificación y control de costes.

- Implementar un sistema para la gestión medioambiental.
- Seleccionar y/o implementar un sistema de información.

- Diseñar y gestionar las operaciones de una organización.

- Analizar el entorno y mercado para la toma de decisiones comerciales de carácter estratégico: comunicación con su entorno, fijación de precios, distribución y desarrollo y gestión de productos y desarrollar estudios de mercado.

- Redactar proyectos de gestión de la innovación tecnológica.

- Planificar y gestionar proyectos.

- Planificar y gestionar la estrategia de una organización.

- Planificar y desarrollar nuevos productos y procesos.

- Evaluar los puestos de trabajo de una organización.

- Diseñar y organizar las instalaciones de una organización.

- Llevar a cabo estudios del impacto de las tecnologías de proceso.

- Planificar las características generales de las tecnologías de proceso.

- Aportar racionalidad al análisis y a la descripción de cualquier problema de la organización.

- Evaluar consecuencias de distintas alternativas de acción y seleccionar las mejores dados los objetivos.

Tabla 3

\section{Competencias específicas o destrezas}

\begin{tabular}{|c|c|c|}
\hline \multicolumn{2}{|c|}{ Destrezas para la organización } & \multirow{2}{*}{$\frac{\text { Destrezas tecnológicas }}{\text { 5I. Diseño y organización de plantas }}$} \\
\hline 39. Asignación óptima de recursos & 45. Planificación de proyectos & \\
\hline 40. Análisis financieros & 46. Planificación estratégica & industriales \\
\hline $\begin{array}{l}\text { 41. Diseño de los sistemas de } \\
\text { información de la empresa }\end{array}$ & $\begin{array}{l}\text { 47. Planificación y control de la } \\
\text { producción }\end{array}$ & $\begin{array}{l}\text { 52. Estudio del impacto de las tecnologías } \\
\text { del proceso }\end{array}$ \\
\hline $\begin{array}{l}\text { 42. Diseño y mejora de procesos } \\
\text { productivos y de servicios }\end{array}$ & $\begin{array}{l}\text { 48. Planificación y desarrollo de nuevos } \\
\text { productos y procesos }\end{array}$ & $\begin{array}{l}\text { 53. Planificar las características generales } \\
\text { de las tecnologías del proceso }\end{array}$ \\
\hline 43. Estudios de mercado & 49. Valoración de puestos de trabajo & 54. Gestión medioambiental \\
\hline 44. Gestión de la innovación tecnológica & 50. Diseño de sistemas organizativos & \\
\hline
\end{tabular}


- Integrarse en la gestión empresarial.

- Identificar las fuentes de información relevante y su contenido.

- Usar habitualmente las tecnologías de la información y comunicaciones en todo su desempeño profesional.

- Comunicarse con fluidez en su entorno y trabajar en equipo.

En la Declaración de Bolonia (1999), Marín-García et al. (2008) y Marín-García et al. (2010) pueden consultarse otras definiciones generales del perfil del egresado del Grado en Ingeniería de Organización Industrial. Además, en las memorias verificadas de los grados en ingeniería de organización industrial que actualmente se imparten en las universidades españolas pueden encontrarse definiciones del perfil del egresado más específicas en función de cada universidad.

\subsection{Los requisitos para la verificación del GIOI de ADINGOR}

La Asociación para el Desarrollo de la Ingeniería de Organización (ADINGOR) es una asociación sin ánimo de lucro española que aglutina a profesionales, docentes e investigadores que desarrollan su labor profesional en el ámbito de la Ingeniería de Organización Industrial. Entre los fines de la asociación se encuentra el de «contribuir al desarrollo de los conocimientos, teóricos y de aplicación práctica, propios del área de Ingeniería de Organización, y en especial, los relativos al diseño, instalación, funcionamiento, gestión, control y mejora de sistemas industriales y de prestación de servicios -tales como empresas y otras organizaciones- integrados por personas, equipos, materiales, energía, información y recursos financieros, orientados a la satisfacción de las necesidades y aspiraciones de los usuarios, teniendo en cuenta las necesidades e intereses de otros implicados y afectados, y de respeto al medioambiente».

De acuerdo con los fines por los que se estableció y ante la falta de una guía común nacional para la elaboración de planes de estudios de GIOl, tal y como sí existía para otros grados de ingeniería (los que capacitaban para el ejercicio de la profesión de ingeniero), ADINGOR desarrolló en 2008 un documento sobre «Requisitos para la verificación del título de Grado en Ingeniería de Organización Industrial» (ADINGOR 2008), con el objeto de transmitir el conocimiento y la experiencia de sus asociados en este ám- bito e influir en los contenidos básicos del mencionado título de Grado, planteando el perfil del egresado, sus capacidades genéricas y específicas y proponiendo una planificación de las enseñanzas.

Según el citado documento, el título de Grado en Ingeniería de Organización Industrial debe capacitar al egresado para la gestión y dirección de empresas industriales y de servicios, así como de instituciones de distinta índole (tanto públicas como privadas; Administración Pública, Universidad, ONGs, empresas consultoras, etc.), en todas sus áreas funcionales: producción, logística, calidad, mantenimiento, compras, comercial, productos, procesos, costes, finanzas, medio ambiente, gestión de la innovación, gestión de proyectos, recursos humanos, prevención de riesgos laborales, responsabilidad social empresarial, etc. Sus capacidades son especialmente adecuadas en actividades con un contenido relevante de proyectos $y / 0$ operaciones en que la tecnología y organización deban interrelacionarse de modo eficaz y eficiente, así como en actividades que impliquen la gestión de la tecnología o de la innovación tecnológica.

Y se identificaban diferentes perfiles profesionales correspondientes al GIOI en relación con las funciones que actualmente desempeñan en la práctica profesional los titulados actuales en esta rama de la ingeniería:

- Dirección general de empresas, especialmente empresas industriales o de servicios con un contenido tecnológico relevante.

- Organización y gestión de la producción y las operaciones.

- Organización y gestión de redes logísticas.

- Gestión de distribución física (almacenes y transportes).

- Gestión de compras y aprovisionamientos.

- Gestión de calidad, seguridad y medio ambiente.

- Gestión de tecnología y de innovación tecnológica.

- Gestión de sistemas de información.

- Gestión de la organización.

- Gestión de recursos humanos.

- Gestión de marketing y comercial.

- Gestión financiera y de costes. 
- Administración pública, especialmente, en áreas de promoción industrial y tecnológica, e I+D+I.

El documento concluía con una recomendación de bloques y competencias para los planes de estudios que se desarrollaran en las universidades españolas (véase Tabla 4).

\section{El Grado en Ingeniería de Organización Industrial en las universidades españolas}

En el curso académico $2011 / 12$ un total de 22 universidades españolas han ofertado el GIOI. En las siguientes secciones se analiza el mapa de la titulación.

\section{I. Mapa de la titulación}

En la actualidad en España hay 23 centros, 9 centros privados y 14 centros públicos, de 22 universidades españolas, 9 universidades privadas y 13 universidades públicas, que imparten, han verificado o están tramitando el título de GIOI (Tabla 5). El GIOI tiene una mayor presencia en el territorio español en aquellas regiones con una considerable tradición industrial (Figura I).

Tabla 4

Bloques y competencias recomendados por ADINGOR para el GIOI

\begin{tabular}{|c|c|c|}
\hline Bloques & $\begin{array}{l}\text { N. }{ }^{\circ} \text { mínimo } \\
\text { de créditos } \\
\text { ECTS }\end{array}$ & Competencias que deben adquirirse \\
\hline $\begin{array}{l}\text { Bloque de Formación. Básica } \\
\text { Deberá incluir las materias } \\
\text { básicas de formación de la } \\
\text { rama de conocimiento. }\end{array}$ & 60 & $\begin{array}{l}\text { Competencias y conocimientos que se adquieren con las materias básicas del } \\
\text { Anexo II del RD de Ordenación de las Enseñanzas Universitarias. }\end{array}$ \\
\hline Tecnologías Industriales & 20 & $\begin{array}{l}\text { Tecnología mecánica. Tecnología de los materiales. Tecnología eléctrica. } \\
\text { Tecnología electrónica. Tecnología de sistemas y automática. Tecnologías } \\
\text { energéticas. Tecnologías medioambientales. }\end{array}$ \\
\hline $\begin{array}{l}\text { Tecnologías de la Información } \\
\text { y las Comunicaciones }\end{array}$ & 16 & $\begin{array}{l}\text { Tecnologías informáticas. Tecnologías de computación. Tecnologías de los } \\
\text { sistemas de información. Redes, sistemas y servicios. }\end{array}$ \\
\hline $\begin{array}{l}\text { Métodos Cuantitativos } \\
\text { y Organización } \\
\text { de la Producción }\end{array}$ & 24 & $\begin{array}{l}\text { Concepto de métodos cuantitativos. Proceso de modelado de sistemas } \\
\text { industriales, económicos y sociales. Algoritmos. Validación. Toma de decisiones. } \\
\text { Optimización. Redes y grafos. Simulación. Teoría de colas. Concepto de } \\
\text { producción y decisiones asociadas. Gestión de stocks. Planificación de la } \\
\text { producción. Estudio del trabajo. Métodos y tiempos. Planificación y gestión de } \\
\text { proyectos. Sistemas de gestión para la dirección. }\end{array}$ \\
\hline $\begin{array}{l}\text { Administración de Empresas } \\
\text { e Ingeniería Económica }\end{array}$ & 20 & $\begin{array}{l}\text { Organización y dirección de empresas. Derecho mercantil y laboral. Técnicas } \\
\text { de control de gestión financiera y de costes. Análisis de inversiones. Finanzas. } \\
\text { Análisis de mercados. Organización del trabajo. Factor humano. Gestión de la } \\
\text { calidad, seguridad y medioambiente. Prevención de riesgos laborales. Sistemas } \\
\text { de información. Gestión integrada ERP. }\end{array}$ \\
\hline $\begin{array}{l}\text { Entorno Industrial } \\
\text { y Tecnológico }\end{array}$ & 16 & $\begin{array}{l}\text { La empresa y el modelo microeconómico. Competitividad estratégica y } \\
\text { estructura del mercado. El entorno y las políticas macroeconómicas. Cambio } \\
\text { tecnológico y estrategia empresarial. Innovación en la empresa. Innovación, } \\
\text { emprender y cultura. El producto: creación y desarrollo de nuevos productos. } \\
\text { Competitividad industrial e innovación. Sistemas regionales y nacionales de } \\
\text { innovación. Política tecnológica y patrones de innovación. }\end{array}$ \\
\hline Proyecto Fin de Grado & 12 & $\begin{array}{l}\text { Ejercicio original a realizar individualmente y presentar y defender ante un } \\
\text { tribunal universitario, consistente en un proyecto de Ingeniería de } \\
\text { Organización Industrial de naturaleza profesional en el que se sinteticen todas } \\
\text { las competencias adquiridas en las enseñanzas. }\end{array}$ \\
\hline
\end{tabular}


Tabla 5

Oferta de plazas del GIOI en los centros universitarios españoles. Fuente: Elaboración propia a partir de los datos del Ministerio de Educación

\begin{tabular}{|c|c|c|c|c|c|c|c|}
\hline Universidad & Acrónimo & Tipo & Centro & Localidad & $\begin{array}{c}\text { Estado } \\
\text { del grado }\end{array}$ & $\begin{array}{c}\text { Plazas ofertadas } \\
201012011\end{array}$ & $\begin{array}{c}\text { Plazas ofertadas } \\
2011 / 2012\end{array}$ \\
\hline $\begin{array}{c}\text { Universidad a } \\
\text { distancia de Madrid }\end{array}$ & UDIMA & Privada & $\begin{array}{c}\text { Facultad de } \\
\text { Enseñanza Técnica }\end{array}$ & Collado Villalba & Grado verificado & - & - \\
\hline $\begin{array}{l}\text { Universidad } \\
\text { Autónoma } \\
\text { de Barcelona }\end{array}$ & UAB & Privada & $\begin{array}{l}\text { Escuela Universitaria } \\
\text { Salesiana de Sarriá }\end{array}$ & Barcelona & Grado Oficial & 80 & 60 \\
\hline Universidad de Burgos & UBU & Pública & $\begin{array}{c}\text { Escuela Politécnica } \\
\text { Superior }\end{array}$ & Burgos & Grado Oficial & - & Sin límite \\
\hline Universidad de Deusto & UDE & Privada & $\begin{array}{c}\text { Facultad } \\
\text { de Ingeniería }\end{array}$ & Bilbao & Grado Official & - & - \\
\hline Universidad de Jaén & UJAEN & Pública & Universidad de Jaén & Jaén & Grado verificado & - & 75 \\
\hline $\begin{array}{l}\text { Universidad de Las } \\
\text { Palmas de Gran } \\
\text { Canaria }\end{array}$ & ULPG & Pública & $\begin{array}{c}\text { Escuela } \\
\text { de Ingenierías } \\
\text { Industriales y Civiles }\end{array}$ & $\begin{array}{c}\text { Las Palmas } \\
\text { de Gran Canaria }\end{array}$ & Grado Oficial & 300 & 70 \\
\hline Universidad de Málaga & UMA & Pública & & Málaga & & & 65 \\
\hline Universidad de Sevilla & US & Pública & CEl Andalucía lech & Sevilla & En trámite & - & 65 \\
\hline $\begin{array}{c}\text { Universidad Europea } \\
\text { de Madrid }\end{array}$ & UEM & Privada & Escuela Politécnica & $\begin{array}{l}\text { Villaviciosa } \\
\text { de Odón }\end{array}$ & Grado Oficial & - & - \\
\hline $\begin{array}{l}\text { Mondragon } \\
\text { Unibertsitatea }\end{array}$ & $M U$ & Privada & $\begin{array}{c}\text { Escuela Politécnica } \\
\text { Superior }\end{array}$ & Mondragon & Grado Official & - & - \\
\hline Universidad de Navarra & UNAV & Privada & $\begin{array}{c}\text { Escuela Superior } \\
\text { de Ingenieros }\end{array}$ & San Sebastián & Grado Official & - & - \\
\hline Universidad del País & & & Escuela Técnica & & & & \\
\hline $\begin{array}{c}\text { Vasco/Euskal Herrico } \\
\text { Unibertsitatea }\end{array}$ & UPV & Pública & $\begin{array}{c}\text { Superior de } \\
\text { Ingeniería de Bilbao }\end{array}$ & Bilbao & Grado Official & 50 & 50 \\
\hline $\begin{array}{c}\text { Universidad Politécnica } \\
\text { de Cartagena }\end{array}$ & UPCT & Pública & $\begin{array}{c}\text { Centro Universitario } \\
\text { de la Defensa }\end{array}$ & $\begin{array}{c}\text { Santiago } \\
\text { de la Ribera }\end{array}$ & Grado Oficial & 71 & 61 \\
\hline $\begin{array}{l}\text { Universidad Politécnica } \\
\text { de Cataluña }\end{array}$ & UPC & Pública & $\begin{array}{c}\text { Escuela de Ingeniería } \\
\text { de Igualada }\end{array}$ & Igualada & Grado Oficial & - & 40 \\
\hline $\begin{array}{l}\text { Universidad Politécnica } \\
\text { de Madrid }\end{array}$ & UPM & Pública & $\begin{array}{l}\text { Escuela Técnica } \\
\text { Sup. de Ingenieros } \\
\text { Industriales }\end{array}$ & Madrid & Grado oficial & - & - \\
\hline $\begin{array}{c}\text { Universitat Politècnica } \\
\text { de València }\end{array}$ & UPVLC & Pública & $\begin{array}{l}\text { Escuela Técnica } \\
\text { Sup. de Ingenieros } \\
\text { Industriales }\end{array}$ & Valencia & Grado oficial & 150 & 100 \\
\hline $\begin{array}{c}\text { Universidad Pontificia } \\
\text { de Salamanca }\end{array}$ & UPSA & Privada & $\begin{array}{l}\text { Escuela Superior } \\
\text { de Ingeniería } \\
\text { y Arquitectura }\end{array}$ & Madrid & Grado Official & * & * \\
\hline $\begin{array}{l}\text { Universidad Rey Juan } \\
\text { Carlos }\end{array}$ & URJC & Pública & $\begin{array}{l}\text { Universidad Rey } \\
\text { Juan Carlos }\end{array}$ & Móstoles & Grado verificado & - & 70 \\
\hline $\begin{array}{l}\text { Universidad } \\
\text { de Valladolid }\end{array}$ & UVA & Pública & $\begin{array}{c}\text { Escuela de Ingenierías } \\
\text { Industriales }\end{array}$ & Valladolid & Grado Oficial & 60 & 50 \\
\hline Universidad de Vic & UVIC & Privada & $\begin{array}{c}\text { Escuela Politécnica } \\
\text { Superior }\end{array}$ & Vic & Grado Official & - & 40 \\
\hline Universidad de Vigo & UVIGO & Pública & $\begin{array}{c}\text { Escuela de Ingeniería } \\
\text { Industrial }\end{array}$ & Vigo & Grado Oficial & 80 & 80 \\
\hline \multirow{2}{*}{$\begin{array}{l}\text { Universidad } \\
\text { de Zaragoza }\end{array}$} & \multirow{2}{*}{ UZAR } & \multirow{2}{*}{ Pública } & $\begin{array}{c}\text { Centro Universitario } \\
\text { de la Defensa }\end{array}$ & Zaragoza & Grado Oficial & 279 & 332 \\
\hline & & & $\begin{array}{c}\text { Escuela Universitaria } \\
\text { Politécnica }\end{array}$ & $\begin{array}{c}\text { La Almunia } \\
\text { de Doña Godina }\end{array}$ & Grado Oficial & 60 & 60 \\
\hline
\end{tabular}

* No impartido hasta la extinción del título actual de IOI. 
Figura I

Mapa de la presencia del GIOI en España

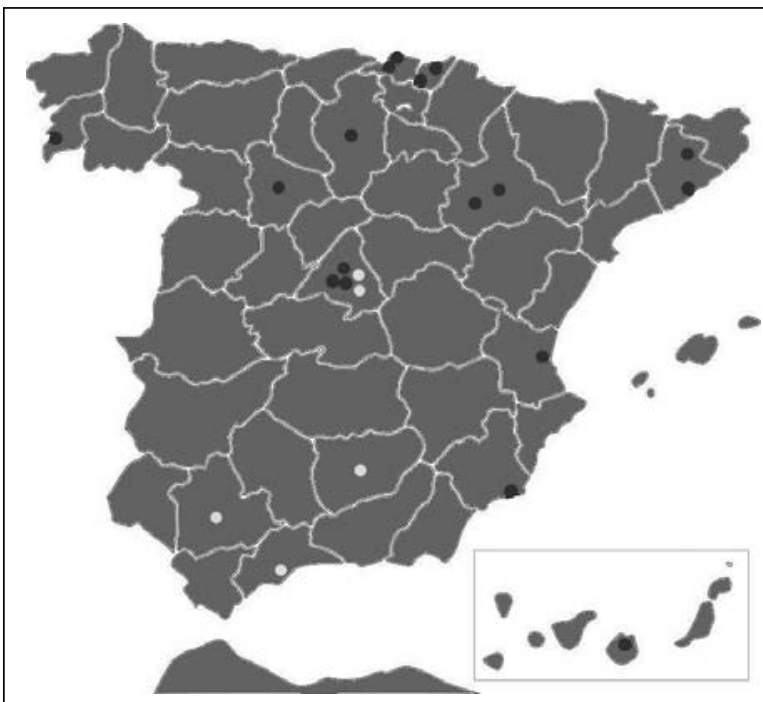

- Grado Oficial Grado verificado/en trámite

La oferta de plazas se aprueba anualmente en el Consejo de Universidades y se publica en el Boletín Oficial del Estado. La Tabla 4 muestra la oferta de plazas, según datos del MED en los centros universitarios españoles donde se imparte la titulación de GIOI. El total de las plazas ofertadas en esta titulación ha sido de I I 30 plazas en el curso 2010/20 I | y de I283 en el curso 20 I I/20 I 2. Los centros con mayor oferta de plazas son el Centro Universitario de la Defensa de Zaragoza adscrito a la Universidad de Zaragoza y la Escuela Técnica Superior de Ingenieros Industriales de la Universitat Politècnica de València.

Cabe destacar como novedad respecto al título de IOI, la existencia de dos centros universitarios del Ministerio de Defensa que han optado por impartir el GIOl. Por otro lado, hasta el momento ningún centro universitario español ofrece este titulo de grado como una doble titulación combinado con otro de ingeniería de la rama industrial.

Con respecto a la demanda satisfecha del GIOI, todavía no están disponibles los datos del curso académico 2010/2011.

\subsection{Comparativa de los planes de estudios propuestos}

Casi la totalidad de las universidades que imparten esta titulación ofrecen acceso público a los planes de estudios en Internet, lo que permite su consulta y análisis. Así, se han comparado aquellos planes de es- tudios cuyos contenidos al completo se encuentran disponibles en la web correspondiente, en total los de 15 centros universitarios (la Universidad de Málaga y la Universidad de Sevilla diseñan de forma conjunta el mismo plan de estudios) de los 23 que aparecen en la Tabla 5 (ver Tabla 8 ).

Tras consultar los planes de estudios que configuran el GIOl en los centros universitarios españoles se ha realizado una comparativa de la estructura general de la titulación y de las asignaturas ofertadas.

\subsubsection{Estructura general de la titulación}

Las enseñanzas conducentes a la obtención del título oficial de GIOI deberán proporcionar una formación adecuada en las bases teóricas y en las tecnologías propias de esta ingeniería (Declaración de Bolonia 1999).

Las directrices generales del Real Decreto I393/2007 indican que los planes de estudios universitarios de grado tendrán una carga lectiva mínima de 240 créditos europeos (ECTS). En el Real Decreto I I 25/2003, de 5 de septiembre, se establece el sistema europeo de créditos y el sistema de calificaciones en las titulaciones universitarias de carácter oficial y validez en todo el territorio nacional.

El Real Decreto I 393/2007 establece que los planes de estudios de grado deberán contener un mínimo de 60 créditos de formación básica. De ellos, al menos 36 estarán vinculados a algunas de las materias que figuran en el anexo II de este Real Decreto para la rama de conocimiento de Ingeniería y Arquitectura, en el caso del GIOl, y deberán concretarse en asignaturas con un mínimo de 6 créditos cada una, que deberán ser ofertadas en la primera mitad del plan de estudios. Los créditos restantes hasta 60 , en su caso, deberán estar configurados por materias básicas de la misma u otras ramas de conocimiento de las incluidas en el anexo II, o por otras materias siempre que se justifique su carácter básico para la formación inicial del estudiante o su carácter transversal. Si se programan prácticas externas, éstas tendrán una extensión máxima de 60 créditos y deberán ofrecerse preferentemente en la segunda mitad del plan de estudios. El trabajo de fin de grado tendrá entre 6 y 30 créditos y deberá realizarse en la fase final del plan de estudios.

En la Tabla 6 se resume la estructura general de los planes de estudios del GIOI analizados en este artículo, en concreto, de aquellas universidades en cuya página web oficial se detalla dicha información. 
Tabla 6

Resumen de la estructura general del GIOI en las universidades españolas

\begin{tabular}{|c|c|c|c|c|c|c|c|c|c|c|c|}
\hline 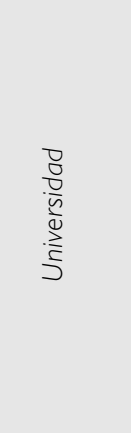 & 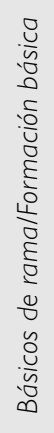 & $\begin{array}{l}\stackrel{\sim}{o} \\
\frac{0}{0} \\
\overrightarrow{0} \\
.00 \\
\overline{0}\end{array}$ & 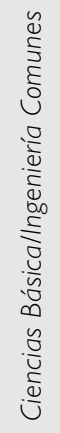 & 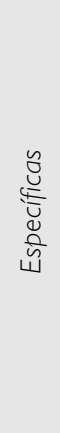 & 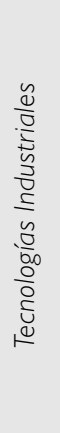 & 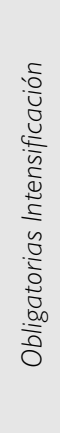 & 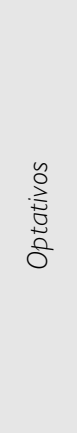 & 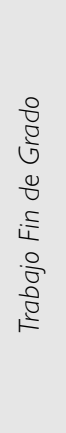 & 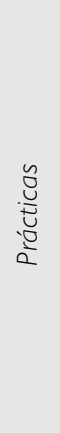 & $\begin{array}{l}\frac{\tilde{U}}{0} \\
\frac{\tilde{J}}{3} \\
\frac{\pi}{3} \\
\tilde{U}\end{array}$ & $\begin{array}{l}\bar{D} \\
\stackrel{0}{0}\end{array}$ \\
\hline UAB & 60 & 120 & & & & & 48 & 12 & & & 240 \\
\hline UBU & 60 & & 72 & 72 & & & 12 & 24 & & & 240 \\
\hline UDE & 60 & 132 & & & & & 30 & 12 & 6 & & 240 \\
\hline UDIMA & 60 & 144 & & & & & 24 & 12 & & & 240 \\
\hline ULPGC & 60 & 150 & & & & & 24 & 6 & & & 240 \\
\hline UMA-US & 60 & & 60 & 60 & & & 30 & 12 & 18 & & 240 \\
\hline$M U$ & 60 & 135 & & & & & 33 & 12 & & & 240 \\
\hline UPV & 48 & 168 & & & & & 18 & 6 & & & 240 \\
\hline UPCT & 60 & 114 & & & & & 22.5 & 12 & 30 & 1.5 & 240 \\
\hline UPVLC & 60 & 121.5 & & & & & 46.5 & 12 & & & 240 \\
\hline UVIC & 66 & 126 & & & & & 30 & 12 & 6 & & 240 \\
\hline UVIGO & 60 & 72 & & 42 & 24 & 24 & 6 & 12 & & & 240 \\
\hline UZAR & 60 & 138 & & & & & 30 & 12 & & & 240 \\
\hline
\end{tabular}

Como puede apreciarse en la tabla anterior, de forma general, y tal como se indica en el Real Decreto 1393/2007, el GIOl cuenta con 60 ECTS de formación básica a excepción de la Escuela Técnica Superior de Ingeniería de Bilbao de la Universidad del País Vasco que oferta 48 y de la Escuela Politécnica Superior de la Universidad de Vic que establece 66.

En cuanto a las materias obligatorias se imparten un promedio de 135, I 4 ECTS aunque algunos planes de estudios se alejan de dicho promedio. En primer lugar, el de la Escuela Politécnica de Superior de Burgos que, en lugar de materias obligatorias, distingue entre materias comunes y específicas con 72 ECTS. El Campus de Excelencia Internacional Andalucía Tech de la Universidad de Málaga y la Universidad de Sevilla establecen 60 ECTS para materias de ciencias básicas de ingeniería y tecnología básica de or- ganización y 60 ECTS para tecnologías específicas de la ingeniería de organización. También, la Escuela de Ingeniería Industrial de la Universidad de Vigo configura 72 ECTS para materias obligatorias comunes de la rama industrial, 42 ECTS para materias específicas de organización industrial, 24 ECTS para tecnologías industriales y 24 ECTS para obligatorias de intensificación.

El promedio de asignaturas optativas ofertadas es de 27,43 ECTS en un rango comprendido entre 12, ofertados por la Escuela Politécnica Superior de Burgos, y 48 ECTS correspondientes a la Escuela Universitaria Salesiana de Sarrià de la Universidad Autónoma de Barcelona.

Respecto al trabajo fin de grado, el promedio de créditos asignados es de 12 ECTS. Por encima de esta media destaca la Escuela Politécnica de Burgos, que 
le otorga 24 ECTS. La mayoría de los centros establecen 12 ECTS excepto la Escuela de Ingenierías Industriales y Civiles de la Universidad de las Palmas de Gran Canaria y la Escuela Técnica Superior de Ingeniería de Bilbao de la Universidad del País Vasco, que han fijado 6 ECTS.

Por otro lado, el Centro Universitario de la Defensa de Cartagena, adscrito de la Universidad Politécnica de Cartagena, asigna un total de 30 ECTS para prácticas y I,5 para actividades culturales. El Campus de Excelencia Internacional Andalucía Tech de la Universidad de Málaga y la Universidad de Sevilla asignan 18 ECTS para prácticas en empresa, movilidad, reconocimiento de créditos y optativas transversales. La Escuela Politécnica Superior de la Universidad de Vic y la Universidad de Deusto establecen también 6 ECTS para prácticas.

\subsubsection{Análisis comparativo de las asignaturas ofertadas}

En esta sección se han comparado las asignaturas impartidas en los planes de estudios analizados sin tener en cuenta las asignaturas optativas. Para ello, se ha utilizado la clasificación de materias propuesta en la Tabla 7.

En la Tabla 8 se muestran los créditos asignados por los diferentes planes de estudios comparados a cada una de las materias de la Tabla 7.

Tabla 7

Clasificación de las materias propuestas en el GIOI

\begin{tabular}{|c|c|c|}
\hline Módulo & Materia & Código materia \\
\hline \multirow{6}{*}{ FORMACIÓN BÁSICA } & Empresa & EMP \\
\hline & Expresión Gráfica & EXG \\
\hline & Física & FIS \\
\hline & Informática & INF \\
\hline & Matemáticas & MAT \\
\hline & Química & QUI \\
\hline \multirow{6}{*}{ TECNOLOGÍAS INDUSTRIALES } & Automática y Electrónica & AUT \\
\hline & Construcciones Industriales & $\mathrm{Cl}$ \\
\hline & Ingeniería Térmica y de Fluídos & ITF \\
\hline & Tecnología Eléctrica & TE \\
\hline & Tecnología Medioambiental & TM \\
\hline & Tecnología Mecánica y Materiales & TMEC \\
\hline \multirow{13}{*}{ ORGANIZACIÓN INDUSTRIAL } & Administración de Empresas & ADM \\
\hline & Calidad & CA \\
\hline & Dirección Comercial & DC \\
\hline & Dirección Estratégica & DE \\
\hline & Dirección Financiera y Contabilidad & DF \\
\hline & Economía General & ECO \\
\hline & Gestión Mantenimiento & GM \\
\hline & Logística & LOG \\
\hline & Métodos Cuantitativos & MC \\
\hline & Organización de Producción & OP \\
\hline & Organización del Trabajo & OT \\
\hline & Proyectos & PR \\
\hline & Sistemas Información & SI \\
\hline \multirow{3}{*}{ TRANSVERSALES } & Técnicas Comunicación & COM \\
\hline & Ciencias Sociales y Humanidades & CS \\
\hline & Idiomas & IDM \\
\hline
\end{tabular}


Tabla 8

Créditos por materia en el GIOI

\begin{tabular}{|c|c|c|c|c|c|c|c|c|c|c|c|}
\hline & \multicolumn{11}{|c|}{ Formación básica } \\
\hline & EMP & \multicolumn{2}{|r|}{$E X G$} & \multicolumn{2}{|l|}{ FIS } & INF & \multicolumn{2}{|c|}{ MAT } & \multicolumn{2}{|l|}{ QUI } & Total \\
\hline$M U$ & 6,0 & \multicolumn{2}{|r|}{6,0} & \multicolumn{2}{|l|}{12,0} & 6,0 & \multicolumn{2}{|c|}{24,0} & 10,5 & \multicolumn{2}{|r|}{64,5} \\
\hline UAB & 6,0 & \multicolumn{2}{|r|}{6,0} & \multicolumn{2}{|l|}{15,0} & 12,0 & \multicolumn{2}{|c|}{21,0} & 6,0 & \multicolumn{2}{|r|}{66,0} \\
\hline UBU & 6,0 & \multicolumn{2}{|r|}{12,0} & \multicolumn{2}{|l|}{12,0} & 6,0 & \multicolumn{2}{|c|}{30,0} & 12,0 & \multicolumn{2}{|r|}{78,0} \\
\hline UDIMA & 6,0 & \multicolumn{2}{|r|}{6,0} & \multicolumn{2}{|l|}{6,0} & 12,0 & & & 12,0 & & 66,0 \\
\hline UEM & 6,0 & & 6,0 & 12,0 & & 6,0 & & & 12,0 & & 66,0 \\
\hline ULPGC & 6,0 & & 6,0 & 12,0 & & 12,0 & & & 18,0 & & 78,0 \\
\hline UMA/US & 6,0 & & 6,0 & 12,0 & & 6,0 & & & 6,0 & & 60,0 \\
\hline UNAV & 18,0 & & 6,0 & 15,0 & & 15,0 & & & 6,0 & & 84,0 \\
\hline UPC & 6,0 & & 0,0 & 6,0 & & 6,0 & & & 6,0 & & 42,0 \\
\hline UPCT & 9,0 & & 6,0 & 6,0 & & 6,0 & & & 10,5 & & 57,0 \\
\hline UPV & 0,0 & & 12,0 & 15,0 & & 6,0 & & & 18,0 & & 90,0 \\
\hline UPVLC & 0,0 & & 6,0 & 15,0 & & 6,0 & & & 10,5 & & 52,5 \\
\hline UVA & 6,0 & & 0,0 & 6,0 & & 6,0 & & & 10,5 & & 58,5 \\
\hline UVIC & 6,0 & & 0,0 & 6,0 & & 18,0 & & & 12,0 & & 60 \\
\hline Promedio & 6,2 & & 5,6 & 10,7 & & 8,8 & & & 10,7 & & 65,9 \\
\hline & & & Tecnc & gías indus & ales & & & & Trans & sales & \\
\hline & AUT & $\mathrm{Cl}$ & ITF & TE & $T M$ & TMEC & Total & COM & CS & IDM & Total \\
\hline$M U$ & 0,0 & 0,0 & 7,5 & 6,0 & 0,0 & 15,0 & 28,5 & 3,0 & 3,0 & 6,0 & 12,0 \\
\hline$\cup A B$ & 7,0 & 0,0 & 6,0 & 7,0 & 3,0 & 12,0 & 35,0 & 3,0 & 12,0 & 3,0 & 12,0 \\
\hline UBU & 12 & 6,0 & 6,0 & 6,0 & 6,0 & 6,0 & 42,0 & 0,0 & 0,0 & 0,0 & 0,0 \\
\hline UDIMA & 6,0 & 0,0 & 6,0 & 112,0 & 6,0 & 24,0 & 54,0 & 0,0 & 12,0 & 6,0 & 18,0 \\
\hline UEM & 12,0 & 0,0 & 12,0 & 6,0 & 0,0 & 12,0 & 42,0 & 6,0 & 6,0 & 6,0 & 18,0 \\
\hline ULPGC & 19,5 & 6,0 & 18,0 & 16,5 & 4,5 & 21,0 & 85,5 & 0,0 & 0,0 & 6,0 & 6,0 \\
\hline UMA/US & 6,0 & 6,0 & 10,5 & 10,5 & 6,0 & 12,0 & 51,0 & 0,0 & 0,0 & 0,0 & 0,0 \\
\hline UNAV & 10,5 & 0,0 & 12,0 & 4,5 & 4,5 & 10,5 & 42,0 & 0,0 & 6,0 & 0,0 & 6,0 \\
\hline UPC & 6,0 & 6,0 & 12,0 & 6,0 & 6,0 & 6,0 & 42,0 & 6,0 & 0,0 & 0,0 & 6,0 \\
\hline UPCT & 4,5 & 0,0 & 7,5 & 4,5 & 3,0 & 9,0 & 5 & 0,0 & 0,0 & 25,5 & 25,5 \\
\hline UPV & 6,0 & 6,0 & 18,0 & 12,0 & 6,0 & 12,0 & 60,0 & 12,0 & 0,0 & 0,0 & 12,0 \\
\hline UPVLC & 9,0 & 0,0 & 13,5 & 4,5 & 0,0 & 9,0 & 36,0 & 0,0 & 0,0 & 0,0 & 0,0 \\
\hline UVA & 13,5 & 0,0 & 10,5 & 6,0 & 6,0 & 15,0 & 51,0 & 0,0 & 0,0 & 0,0 & 0,0 \\
\hline UVIC & 18,0 & 6,0 & 6,0 & 6,0 & 0,0 & 6,0 & 42,0 & 0,0 & 0,0 & 0,0 & 0,0 \\
\hline Promedio & 9,3 & 2,6 & 10,4 & 7,7 & 3,6 & $|2|$, & 45,7 & 1,9 & 2,8 & 3,5 & 8,3 \\
\hline
\end{tabular}

\begin{tabular}{|c|c|c|c|c|c|c|c|c|c|c|c|c|c|c|}
\hline & \multicolumn{14}{|c|}{ Organización industrial } \\
\hline & $A D M$ & $C A$ & $D C$ & $D E$ & $D F$ & ECO & $G M$ & LOG & $M C$ & $O P$ & OT & $P R$ & SI & Total \\
\hline $\mathrm{MU}$ & 0,0 & 12,0 & 4,5 & 10,5 & 3,0 & 0,0 & 0,0 & 12,0 & 6,0 & 1,0 & 3,0 & 6,0 & 6,0 & 63,0 \\
\hline$\cup A B$ & 6,0 & 6,0 & 0,0 & 18,0 & 6,0 & 6,0 & 0,0 & 0,0 & 15,0 & 7,0 & 0,0 & 0,0 & 0,0 & 64,0 \\
\hline UBU & 0,0 & 6,0 & 6,0 & 6,0 & 6,0 & 0,0 & 6,0 & 6,0 & 12,0 & 18,0 & 6,0 & 6,0 & 0,0 & 78,0 \\
\hline UDIMA & 0,0 & 6,0 & 6,0 & 6,0 & 12,0 & 12,0 & 0,0 & 6,0 & 12,0 & 12,0 & 6,0 & 12,0 & 6,0 & 96,0 \\
\hline UEM & 0,0 & 6,0 & 6,0 & 6,0 & 6,0 & 0,0 & 0,0 & 6,0 & 12,0 & 12,0 & 0,0 & 12,0 & 6,0 & 72,0 \\
\hline ULPGC & 0,0 & 6,0 & 6,0 & 18,0 & 6,0 & 6,0 & 0,0 & 0,0 & 6,0 & 6,0 & 6,0 & 6,0 & 6,0 & 72,0 \\
\hline UMA/US & 10,5 & 6,0 & 4,5 & 4,5 & 0,0 & 0,0 & 6,0 & 0,0 & 10,5 & 13,5 & 10,5 & 4,5 & 4,5 & 75,0 \\
\hline UNAV & 0,0 & 6,0 & 3,0 & 3,0 & 6,0 & 0,0 & 0,0 & 4,5 & 10,5 & 9,0 & 6,0 & 3,0 & 19,5 & 70,5 \\
\hline UPC & 0,0 & 0,0 & 6,0 & 6,0 & 6,0 & 0,0 & 0,0 & 6,0 & 12,0 & 6,0 & 12,0 & 0,0 & 6,0 & 60,0 \\
\hline UPCT & 0,0 & 0,0 & 0,0 & 6,0 & 0,0 & 0,0 & 0,0 & 0,0 & 4,5 & 9,0 & 9,0 & 4,5 & 0,0 & 33,0 \\
\hline UPV & 0,0 & 6,0 & 6,0 & 18,0 & 12,0 & 6,0 & 0,0 & 0,0 & 12,0 & 6,0 & 18,0 & 6,0 & 6,0 & 96,0 \\
\hline UPVLC & 4,5 & 4,5 & 4,5 & 4,5 & 9,0 & 0,0 & 0,0 & 0,0 & 9,0 & 19,5 & 9,0 & 6,0 & 6,0 & 76,5 \\
\hline UVA & 10,5 & 0,0 & 0,0 & 16,5 & 6,0 & 0,0 & 0,0 & 0,0 & 10,5 & 12,0 & 0,0 & 0,0 & 10,5 & 66,0 \\
\hline UVIC & 0,0 & 6,0 & 6,0 & 6,0 & 6,0 & 0,0 & 0,0 & 0,0 & 12,0 & 12,0 & 6,0 & 0,0 & 0,0 & 54,0 \\
\hline Promedio & 2,3 & 5,0 & 4,2 & 9,2 & 6,0 & 2,1 & 0,9 & 2,9 & 10,3 & 10,1 & 6,5 & 4,7 & 5,5 & 69,7 \\
\hline
\end{tabular}


En cuanto a la formación básica, se asignan un promedio de 65,9 ECTS destacando los 90 ECTS establecidos por la en la Escuela Técnica Superior de Ingeniería de Bilbao y los 42 ECTS de la Escuela Politécnica Superior de la Universidad de Vic. Las matemáticas son la materia que tiene asignado un mayor número de créditos, con un valor promedio de 23,9 ECTS, cabe resaltar los 39 ECTS establecidos en la Escuela Técnica Superior de Ingeniería de Bilbao, seguidos por los 30 ECTS de la Escuela Politécnica Superior de la Universidad de Burgos y la Escuela Técnica Superior de Ingenierías Industriales de la Universidad de Valladolid. Por debajo del valor promedio, cabe señalar los 15 ECTS impartidos en la Escuela Técnica Superior de Ingenieros Industriales de la UPVLC, los I 8 ECTS asignados en la Escuela Politécnica Superior de la Universidad de Vic y la Escuela de Ingeniería de Igualada perteneciente a la Universidad Politécnica de Cataluña. Las materias de física y química siguen a las matemáticas con una asignación media de 10,7 ECTS. En cuanto a física, existen cinco centros universitarios que únicamente asignan 6 ECTS, por debajo del valor promedio de la materia, mientras que otros cuatro ofrecen 15 ECTS. Respecto a química, cuatro centros únicamente asignan 6 ECTS, mientras que dos ofertan un total de 18 ECTS para dicha materia. La materia de informática tiene asignada una media de 8,8 ECTS destacando los 18 créditos establecidos en la Escuela Politécnica Superior de la Universidad de Vic, seguida de los I5 ECTS definidos en la Escuela Superior de Ingenieros de la Universidad de Navarra. Existen nueve centros que ofertan únicamente 6 ECTS, por debajo del valor promedio. La materia de empresa implica un promedio de 6,2 ECTS impartidos, aunque tanto en la Escuela Técnica Superior de Ingeniería de Bilbao y la Escuela Técnica Superior de Ingenieros Industriales de la UPVLC no se ha definido tal materia; de forma contraria, en la Escuela Superior de Ingenieros de la Universidad de Navarra se han establecido I 8 ECTS y en el Centro Universitario de Defensa de la Universidad Politécnica de Cartagena se han definido 9 ECTS. El resto de universidades han establecido 6 ECTS para esta materia de empresa. Por último, en el caso de expresión gráfica se ha establecido un promedio de 5,6 ECTS, con tres centros que no han asignado ningún crédito a esta materia. Por el contrario, la Escuela Politécnica Superior de la Universidad de Burgos y la Escuela Técnica Superior de Ingeniería de Bilbao establecen 12 ECTS. El resto de las universidades estudiadas asignan 6 ECTS para esta materia de expresión gráfica.
Con respecto a las tecnologías industriales, se asignan una media de 45,7 ECTS. En los extremos, la Escuela de Ingenierías Industriales y Civiles de la ULPGC define un total de 85,5 créditos, seguida de la Escuela Técnica Superior de Ingeniería de Bilbao con 60 ECTS y la Facultad de Enseñanza Técnica de la Universidad a distancia de Madrid. Por debajo de la media, estarían todas las demás, cabe destacar la Escuela Politécnica Superior de la Mondragón Unibertsitatea y el Centro universitario de la defensa de Cartagena con 28,5 créditos, seguidas de la Escuela Universitaria Salesiana de Sarrià de la Universidad Autónoma de Barcelona con 35 ECTS. En cuanto a las materias, la tecnología mecánica y de materiales, la teoría de fluidos y energética y la automática y electrónica son las que cuentan con un número mayor de créditos frente a las construcciones industriales, la tecnología medioambiental y la tecnología eléctrica.

En cuanto a las materias transversales cabe destacar los 25,5 ECTS asignados a idiomas por el Centro universitario de la defensa Cartagena, los 12 ECTS asignados a técnicas de comunicación por la EscuelaTécnica Superior de Ingeniería de Bilbao y los 12 establecidos para ciencias sociales y humanidades por la Escuela Universitaria Salesiana de Sarriá de la UAB y la Facultad de Enseñanza Técnica de la Universidad a distancia de Madrid.

Relacionado específicamente con la organización industrial, se asignan una media de 69,7 ECTS. En los extremos, la Escuela Técnica Superior de Ingeniería de Bilbao y la Facultad de Enseñanza Técnica de la Universidad a distancia de Madrid imparten un total de 96 ECTS, la Escuela Politécnica Superior de la Universidad de Burgos con 78 ECTS y la Escuela Técnica Superior de Ingenieros Industriales de la Universitat Politècnica de València con 76,5 ECTS. Por debajo de la media, cabe destacar el Centro universitario de la defensa de Cartagena que establece 33 créditos, seguido de la Escuela Politécnica Superior de la Universidad de Vic con 54 ECTS y la Escuela de Ingeniería de Igualada de la Universidad Politécnica de Cataluña con 60 ECTS. En cuanto a las materias, los métodos cuantitativos, la organización de la producción y la dirección estratégica y son las que cuentan con un número mayor de créditos frente a la gestión del mantenimiento, la economía general, la administración de empresas y la logística. En un término medio estarían la organización del trabajo, la dirección financiera, los sistemas de información, la dirección comercial, la calidad y proyectos. La Figura 2 resume para cada universidad la distribución de las 
tipologías de las materias de organización industrial. La tipología PRO aglutina las materias de OP, OT y LOG. La tipología ECO considera ECO más ADE. La tipología ADE incluye DE y ADM. El resto de tipología se corresponde únicamente con la materia de igual nombre.

La Figura 2 pone de manifiesto que, en general, PRO es la tipología de materias que aglutina más créditos, aunque con excepciones en la UAB, la UVA y la ULPGC donde ADE la supera; también pone de manifiesto la importancia de MC. Además, se refleja como la UNAV es la que se adapta en mayor medida a los requisitos de ADINGOR descritos en la sección 3.4. La Tabla 9 compara las universidades en función de la agrupación de tipologías de materias PRO+MC y ECO+ADE. Hay 3 universidades (UBA, ULPGC y UVA) que otorgan un mayor peso a $\mathrm{ADE}+\mathrm{ECO}$, mientras que el resto se lo otorgan a $\mathrm{PRO}+\mathrm{MC}$ de una manera similar excepto los 2 extremos (UBU y MU).
Tabla 9

Universidades por tipologías de materias en el GIOI

\begin{tabular}{cccc}
\cline { 2 - 4 } & $P R O+M C$ & $A D E+E C O$ & dif \\
\hline UBU & 42 & 12 & 30 \\
UPC & 36 & 12 & 24 \\
UNAV & 30 & 9 & 21 \\
UPVLC & 37,5 & 18 & 19,5 \\
UMA/US & 34,5 & 15 & 19,5 \\
UEM & 30 & 12 & 18 \\
UVIC & 30 & 12 & 18 \\
UPCT & 22,5 & 6 & 16,5 \\
MU & 21 & 13,5 & 7,5 \\
UDIMA & 36 & 30 & 6 \\
UPV & 36 & 36 & 0 \\
UVA & 22,5 & 33 & $-10,5$ \\
ULPGC & 18 & 30 & -12 \\
UAB & 22 & 36 & -14 \\
\hline
\end{tabular}

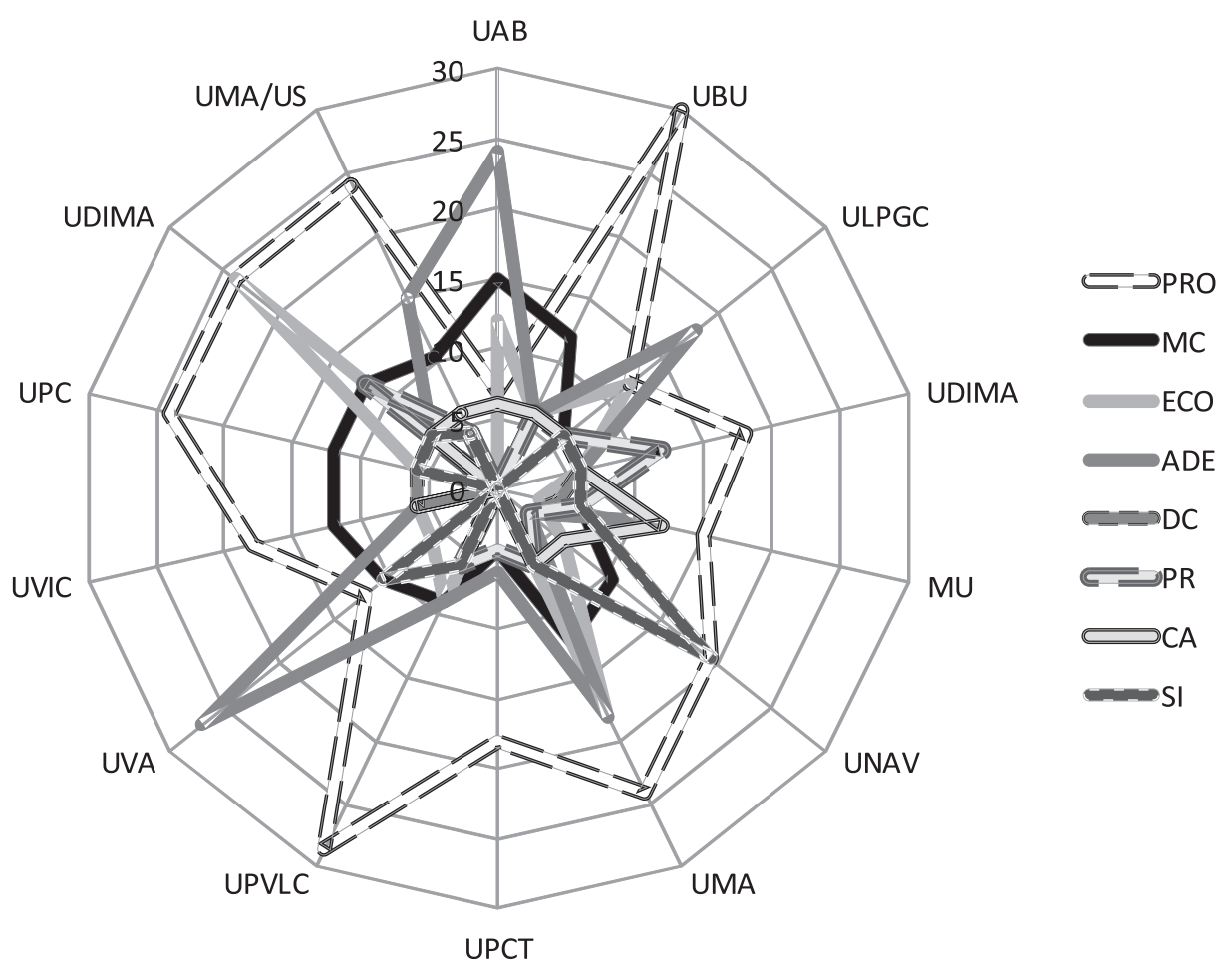




\section{Conclusiones}

En este artículo se ha analizado el diseño de los planes de estudios en las universidades españolas del GIOI. Para ello, previamente se han revisado los antecedentes históricos de esta titulación que se remontan al siglo XIX. En la actualidad y hasta su extinción, prevista para el curso académico 20 I 3/20 I4, coexistirán el GIOl y la titulación de $2^{\circ}$ ciclo de $|O|$ (plan de estudios de 1992). A continuación, se han definido las enseñanzas del $\mathrm{GlOl}$ en cuanto a los objetivos de la titulación, el perfil del ingresado y las competencias del perfil del egresado. En este punto se han revisado las recomendaciones de ADINGOR al respecto de los requisitos para la verificación del GIOl.

En la actualidad, hay 23 centros de 22 universidades españolas que imparten, han verificado o están tramitando el GIOl. De entre éstos, se han analizado los planes de estudios completos y disponibles en Internet de 15 centros universitarios.

De forma resumida, los planes se componen de 60 ECTS de formación básica con un mayor número de créditos asignados a matemáticas, física y química. Además, se contempla el aprendizaje-enseñanza de tecnologías industriales, con un promedio de 45,7 ECTS, con una mayor carga de créditos asignados a la tecnología mecánica y de materiales, la teoría energética y mecánica de fluidos y la automática y electrónica. En cuanto a la formación específica de organización industrial, con un promedio de 69,7 ECTS, los métodos cuantitativos, la organización de la producción y la dirección estratégica son las que cuentan con un número mayor de créditos. Por tipologías de materias, de forma extendida se otorga un mayor número de créditos a PRO (organización de la producción, organización del trabajo y logística) + MC (métodos cuantitativos), lo que se adapta a la propuesta de ADINGOR.

\section{Bibliografía}

ADINGOR, 2008, Documento de Requisitos para la verificación del título de «Grado en Ingeniería de Organi- zación Industrial». Boletín de la Asociación para el Desarrollo de la Ingeniería de Organización, 2, pp. I0-I5.

ANECA, 2006, Libro blanco del título de Grado en Ingeniero de Organización Industrial (Management Engineering). Agencia Nacional de Evaluación de la Calidad y Acreditación.

BARNETT, R., 200 I, Los límites de la competencia. El conocimiento, la educación, la sociedad,, Barcelona: Gedisa.

Declaración de Bolonia, 1999, The European Higher Education Area. Declaración conjunta de los Ministros Europeos de Educación.

FIGUERA, J., 2007, Análisis del pasado de la Ingeniería de Organización en España para mantener su competitividad en el futuro. I International Conference on Industrial Engineering and Industrial Management. Madrid, pp. $931-940$

LASNIER, F., 2000, Réussir la formation par compétences, Montreal: Guérin.

MAFFIOLI, F.y AUGUSTI, G., 2003, Tuning Engineering Education into the European Higher Education Orchestra. European Journal of Engineering Education, 28 (3), pp. 25 I-73.

MARÍN-GARCÍA, J., GARCIA-SABATER, J. P. y CANÓSDARÓS, L., 20 I 0, La Ingeniería de Organización ante el diseño de los nuevos títulos europeos. Dirección y Organización, 41, pp.35-43.

MARÍN-GARCÍA, J., GARCÍA-SABATER, J. P., MIRALLES, C. y RODRÍGUEZVILLALOBOS, A., 2008, La Ingeniería de Organización en un entorno educativo globalizado: reflexiones y propuestas para la universidad española. II International Conference on Industrial Engineering and Industrial Management. Burgos, pp. 817-826.

MINISTERIO DE EDUCACIÓN, CULTURAY DEPORTE, 2003, La integración del sistema universitario español en el Espacio Europeo de Educación Superior. Documento-Marco.

SOLANA, T., 2009, La gestión por competencias en las ingenierías.

UNIVERSIDAD DE DEUSTO, 2000, Competencias, (Documento interno inédito), 